\title{
Malignant Phyllodes Tumor Presenting in Bone, Brain, Lungs, and Lymph Nodes
}

\author{
Eric D. Johnson $^{a} \quad$ Evin Gulbahce $^{b} \quad J o s e p h M^{\prime} N a l l y{ }^{c}$ Saundra S. Buys ${ }^{d}$ \\ ${ }^{a}$ Department of Internal Medicine, University of Utah School of Medicine,

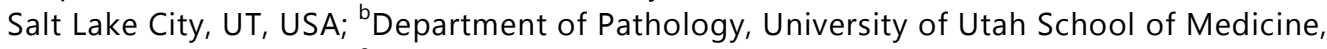 \\ Salt Lake City, UT, USA; 'Department of Radiology, University of Utah School of Medicine, \\ Salt Lake City, UT, USA; ${ }^{d}$ Huntsman Cancer Institute at the University of Utah School of \\ Medicine, Salt Lake City, UT, USA
}

\section{Keywords}

Breast cancer · Phyllodes tumor · Phyllodes sarcoma - Metastasis · Recurrence

\begin{abstract}
Introduction: Phyllodes tumors (PTs) are rare fibroepithelial tumors of the breast which are classified as benign, borderline, or malignant. Malignant PTs account for $<1 \%$ of malignant breast tumors, and borderline tumors have potential to progress to malignant tumors. Metastatic recurrences are most commonly documented in bone and lungs. We report an extremely rare presentation of recurrent malignant PTs involving the brain, lung, lymph nodes, and bone. Case: A 66-year-old female presented with a large breast mass. Biopsy identified malignant PT, treated by mastectomy. One year later she presented with acute back pain; imaging showed pathological L4 spinal compression fracture. Core biopsy confirmed PT. Staging identified additional metastases in the lymph nodes, brain, and lung. Discussion: PTs are rare and fast-growing tumors that originate from periductal stromal tissues and are composed of both epithelial and stromal components. Histologically, they are classified as benign, borderline, or malignant. The prognosis of the malignant type is poorly defined, with local recurrence occurring in $10-40 \%$ and metastases in $10 \%$. Chemotherapy and radiotherapy are generally ineffective in this tumor type. The most common metastatic sites for malignant cases are the lung and bones, but in rare instances, PTs may metastasize elsewhere.
\end{abstract}


Conclusion: We report a rare presentation of recurrent malignant PT presenting as pathological fracture of the lumbar spine with impingement on the spinal column, along with cerebellar, nodal, and pulmonary metastases. Only 1 similar case has been previously reported.

(C) 2016 The Author(s)

Published by S. Karger AG, Basel

\section{Introduction}

Phyllodes tumors (PTs) are rare fibroepithelial malignancies of the breast and account for $<1 \%$ of all malignant breast tumors [1]. They are rapidly growing tumors that originate from the periductal stroma and are comprised of both epithelial and stromal histological tissues [2]. PTs are classified by histopathological features as either benign, borderline, or malignant [3]. All PTs have the potential to become malignant; however, behaviorally this is relatively poorly predicted from histological appearance $[4,5]$. PTs are usually solitary tumors, but can be associated with other malignancies of the breast [6]. In particular, malignant differentiation of both the epithelial and stromal components of PTs can occur, with carcinoma, both invasive and in situ, arising from the epithelial component, and sarcomas such as chondrosarcoma, osteosarcoma and liposarcoma in association with the stromal component [4]. PTs can also coexist with a distinctly separate malignant tumor in either the ipsilateral and/or contralateral breast [7]. We present a case of malignant PT, treated with simple mastectomy 1 year prior, presenting with a pathological L4 compression fracture with evidence of metastases in the brain, lung, and L4 paraspinal region. The case was complicated by progressive unilateral lower extremity sensory and motor loss, requiring surgical resection and lumbar spinal fusion.

\section{Case}

A 66-year-old woman with a history of pulmonary embolism in 2004, Factor V Leiden (heterozygote for trait), severe cellulitis of left leg in 2006 resulting in left lower extremity lymphatic insufficiency, benign essential hypertension, obstructive sleep apnea with nightly continuous positive airway pressure usage, and partial thyroidectomy in 1996 for multinodular goiter. Complete thyroid resection in 2006 for recurrent thyroid nodules identified an unsuspected papillary thyroid cancer. She underwent treatment with radioactive iodine and had no evidence of residual or recurrent disease. She presented to her primary care physician regarding a mass in her left breast that had grown rapidly over several months. Mammography in July 2014 showed an irregular mass measuring $15.5 \mathrm{~cm} \mathrm{AP} \times 16.3 \mathrm{~cm} \mathrm{ML} \times$ $12.6 \mathrm{~cm} \mathrm{CC}$, with circumscribed margins in the central region of the breast (Fig. 1). Ultrasound demonstrated a hypervascular complex cystic and solid mass with circumscribed margins in the central region replacing nearly the entire breast. A core biopsy showed a fibroepithelial neoplasm with stromal atypia and marked hypercellularity (Fig. 2a). All cytokeratin stains and p63 were negative in the stroma, ruling out metaplastic carcinoma; the differential diagnosis included periductal stromal sarcoma and PT. Systemic staging with chest CT showed no evidence of metastatic disease. Excision of the tumor followed by mastectomy confirmed malignant PT, $16 \mathrm{~cm}$ in greatest dimension, with stromal hypercellularity, nuclear pleomorphism, $>10$ mitoses per 10 high-power fields, necrosis, and infiltrative margins (Fig. 2b). Both classic and pleomorphic lobular carcinoma in situ were also seen. The margins were free. The skin and nipple were negative for tumor. 


\section{Case Reports in Oncology}

The patient was well for 1 year, but presented to the emergency department in October 2015 with sudden intense back pain, accompanied by immediate weakness and loss of sensation in her left leg. Imaging showed a pathological compression fracture of the L4 vertebrae (Fig. 3). Core biopsy of the paraspinal lesion showed spindle cell neoplasm consistent with metastatic PT (Fig. 4). Further staging showed pulmonary parenchymal disease and hilar/mediastinal lymphadenopathy (Fig. 5) and a cerebellar metastasis (Fig. 6) in addition to the lesion in the lumbar spine. Metastatic thyroid cancer to these locations was considered, but felt to be unlikely due to the low likelihood of metastatic papillary thyroid cancer in this setting and the biopsy-proven phyllodes metastases.

Despite the use of high-dose steroids and radiation therapy (8 Gy in 1 fraction), the patient noted progressive weakness and numbness of the left lower extremity, without change in bowel or bladder function. She underwent surgery with tumor debulking and lumbar spinal fusion. Pathology showed spindle cell sarcoma similar to her prior malignant PT morphologically and immunohistochemically.

\section{Discussion}

PTs express a wide range of clinical, histological, and cytological features [1]. Based on histological and cytological findings, PTs are classified as benign, borderline, or malignant [2, $5,6]$. This classification allows for the interpretation of PTs as a continuum on a spectrum of fibroepithelial neoplasms of breast tissue; benign PTs are difficult to distinguish from fibroadenomas, while malignant PTs can grow in size quickly and metastasize early $[5,8,9]$. Microscopically, PTs are characterized by a leaf-like appearance, related to projections of hypercellular stroma into epithelium-lined cystic spaces. The presence of a dual population of both epithelial and stromal cells is necessary for the diagnosis of PT. There are numerous histological grading systems for PTs, with most being 3-tiered - "benign, borderline, or malignant" or "low, intermediate, or high grade" - and use the same histological parameters (with varying cutoffs): margin characteristics, cellular atypia, stromal overgrowth, stromal cellularity, and mitotic rate [3]. A variety of associated malignancies can arise from PTs with their dual population of cells [2]. The stromal cells can demonstrate sarcomatous differentiation, while the epithelial component can become malignant with ductal/lobular carcinoma in situ or invasive carcinoma [4]. There is insufficient experience to clearly define the most appropriate therapy $[8,10,11]$, but wide local excision with a margin of $1 \mathrm{~cm}$ is recommended, with no recommendation for chemotherapy or hormone therapy $[5,12]$. However, some authors suggest the use of adjuvant radiotherapy in PTs $>5 \mathrm{~cm}$ with $>20$ mitoses per 10 high-power fields, as these tumors may have a higher local recurrence rate [13].

The prognosis of PTs is variable, with local recurrence rates ranging from 10 to $40 \%$ (average 15\%) and distant metastases occurring in 10\% of all PTs and up to $20 \%$ of malignant PTs $[1,14]$. Survival after metastatic disease is poor, with various case series reporting median survival ranging from 4 to 17 months, with large variability based on the site of metastatic disease $[4,9,15]$. Other large prospective studies have shown 5-year disease-free survival rates of $96 \%$ in benign PTs and of $66 \%$ in malignant PTs [4]. Our case was complicated by acute neurologic symptoms accompanying pathological fracture of a vertebral body and evidence of metastasis to distant sites. It was decided that local wide excision, placement of orthopedic hardware, and close surveillance of the metastatic lesions would be the best treatment course for this unique case $[8,12,14]$. 
Johnson et al.: Malignant Phyllodes Tumor Presenting in Bone, Brain, Lungs, and Lymph Nodes

In summary, we report an extremely rare presentation of malignant PT with metastatic disease in the brain, lung, and lumbar spine with epidural extension. This case demonstrates the need for further research into the most appropriate management of metastatic PT.

\section{Statement of Ethics}

The patient's informed consent was acquired prior to publication of the case.

\section{Disclosure Statement}

The authors have no disclosures to declare.

\section{References}

1 Telli M, Horst K, Guardino A, Dirbas F, Carlson R: Phyllodes tumors of the breast: natural history, diagnosis, and treatment. J Natl Compr Canc Netw 2007;5:324-330.

2 Lindquist K, van Heerden J, Weiland L, Martin J: Recurrent and metastatic cystosarcoma phyllodes. Am J Surg 1982;144:341-343.

-3 Hassouna J, Damak T, Gamoudi A, Chargui R, Khomsi F, Mahjoub S, Slimene M, Dhiab T, Hechiche M, Boussen H, Rahal K: Phyllodes tumors of the breast: a case series of 106 patients. Am J Surg 2006;192: 141-147.

-4 Yabanoğlu H, Colakoglu T, Aytac HO, Parlakgumus A, Bolat FA, Pourbagher A, Yildirim S: Comparison of predictive factors for the diagnosis and clinical course of phyllodes tumours of the breast. Acta Chir Belg 2015;115:27-32.

5 Kim S, Kim J, Kim D, Jung W, Koo J: Analysis of phyllodes tumor recurrence according to the histologic grade. Breast Cancer Res Treat 2013;141:353-363.

-6 Akcakaya A, Erozgen K, Memmi N, Hot S, Yildiz M, Gucin Z, Adas G, Kemik O: Unpredictable behavior of breast phyllodes tumors. Eur Rev Med Pharmacol Sci 2014;18:2655-2661.

7 Wang $\mathrm{H}$, Wang X, Wang C: Comparison of clinical characteristics between benign borderline and malignant phyllodes tumors of the breast. Asian Pac J Cancer Prev 2014;15:10791-10795.

$>8$ Cheng S, Chang Y, Liu TP, Lee JJ, Tzen CY, Liu CL: Phyllodes tumor of the breast: the challenge persists. World J Surg 2006;30:1414-1421.

-9 Grabowski J, Salzstein S, Sadler G, Blair S: Malignant phyllodes tumors: a review of 752 cases. Am Surg 2007;73:967-969.

-10 Jones A, Rizzolo S, Cotler J, Star A, Slemmer R: Metastatic cystosarcoma phyllodes associated with paraplegia: an uncommon complication of an uncommon tumor. J Spinal Disord 1993;6:71-75.

11 Muller K, Tafe L, Abreu F, Peterson J, Wells W, Barth R, Marotti J: Benign phyllodes tumor of the breast recurring as a malignant phyllodes tumor and spindle cell metaplastic carcinoma. Hum Pathol 2015;46: 327-333.

12 Asoglu O, Ugurlu M, Blanchard K, Grant C, Reynolds C, Cha S, Donohue J: Risk factors for recurrence and death after primary surgical treatment of malignant phyllodes tumors. Ann Surg Oncol 2004;11: 1011-1017.

13 Rowe J, Prayson R: Metastatic malignant phyllodes tumor involving the cerebellum. J Clin Neurosci 2015;22:226-227.

14 Chaney A, Pollack A, McNeese M, Zagars G, Pisters P, Pollock R, Hunt K: Primary treatment of cystosarcoma phyllodes of the breast. Cancer 2000;89:1502-1511.

15 McGregor G, Knowling M, Este F: Sarcoma and cystosarcoma phyllodes tumors of the breast a retrospective review of 58 cases. Am J Surg 1994;167:477-480. 


\section{Case Reports in Oncology}

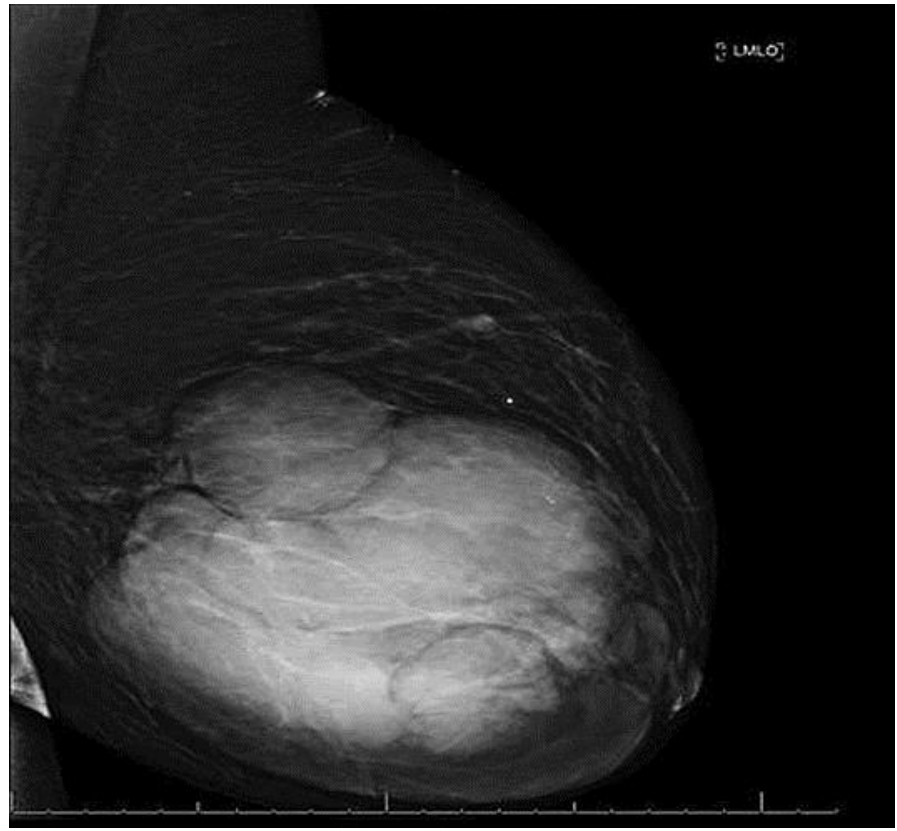

Fig. 1. Mammogram of the left breast. There is a high-density, irregular mass measuring $15.5 \mathrm{~cm} \mathrm{AP} \times$ $16.3 \mathrm{~cm} \mathrm{ML} \times 12.6 \mathrm{~cm} \mathrm{CC}$, with circumscribed margins in the central region of the breast.

Johnson et al.: Malignant Phyllodes Tumor Presenting in Bone, Brain, Lungs, and Lymph Nodes 


\section{Case Reports in Oncology}

\begin{tabular}{l|l}
\hline Case Rep Oncol 2016;9:861-868 \\
\hline DOI: 10.1159/000453660 & $\begin{array}{l}\text { C 2016 The Author(s). Published by S. Karger AG, Basel } \\
\text { www.karger.com/cro }\end{array}$ \\
\hline
\end{tabular}

Johnson et al.: Malignant Phyllodes Tumor Presenting in Bone, Brain, Lungs, and Lymph Nodes

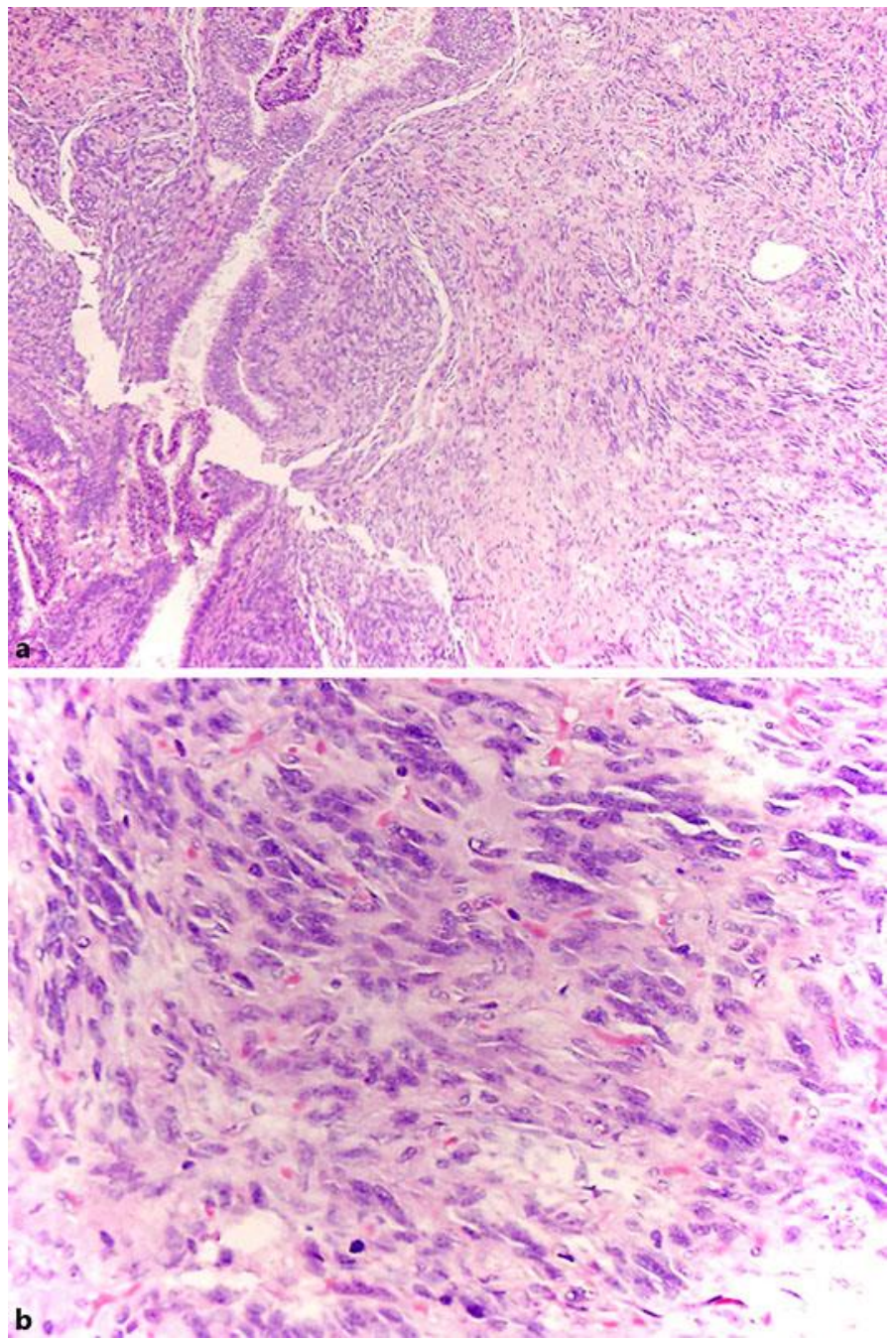

Fig. 2. Breast pathology. a H\&E stain of the breast biopsy showing fibroepithelial neoplasm composed of glandular epithelium with proliferative hyperplasia on the background of a markedly hypercellular spindle cell stroma. Faint characteristic leaf-like architecture and stromal overgrowth are seen. $\times 10$. $\mathbf{b}$ H\&E stain of the breast excision showing fibroepithelial stromal hypercellularity, nuclear pleomorphism, $>10$ mitoses per 10 high-power fields, necrosis, and infiltrative margins consistent with malignant phyllodes tumor. $\times 40$. 


\section{Case Reports in Oncology}

\begin{tabular}{l|l}
\hline Case Rep Oncol 2016;9:861-868 \\
\hline DOI: 10.1159/000453660 & $\begin{array}{l}\text { C } 2016 \text { The Author(s). Published by S. Karger AG, Basel } \\
\text { www.karger.com/cro }\end{array}$ \\
\hline
\end{tabular}

Johnson et al.: Malignant Phyllodes Tumor Presenting in Bone, Brain, Lungs, and Lymph Nodes
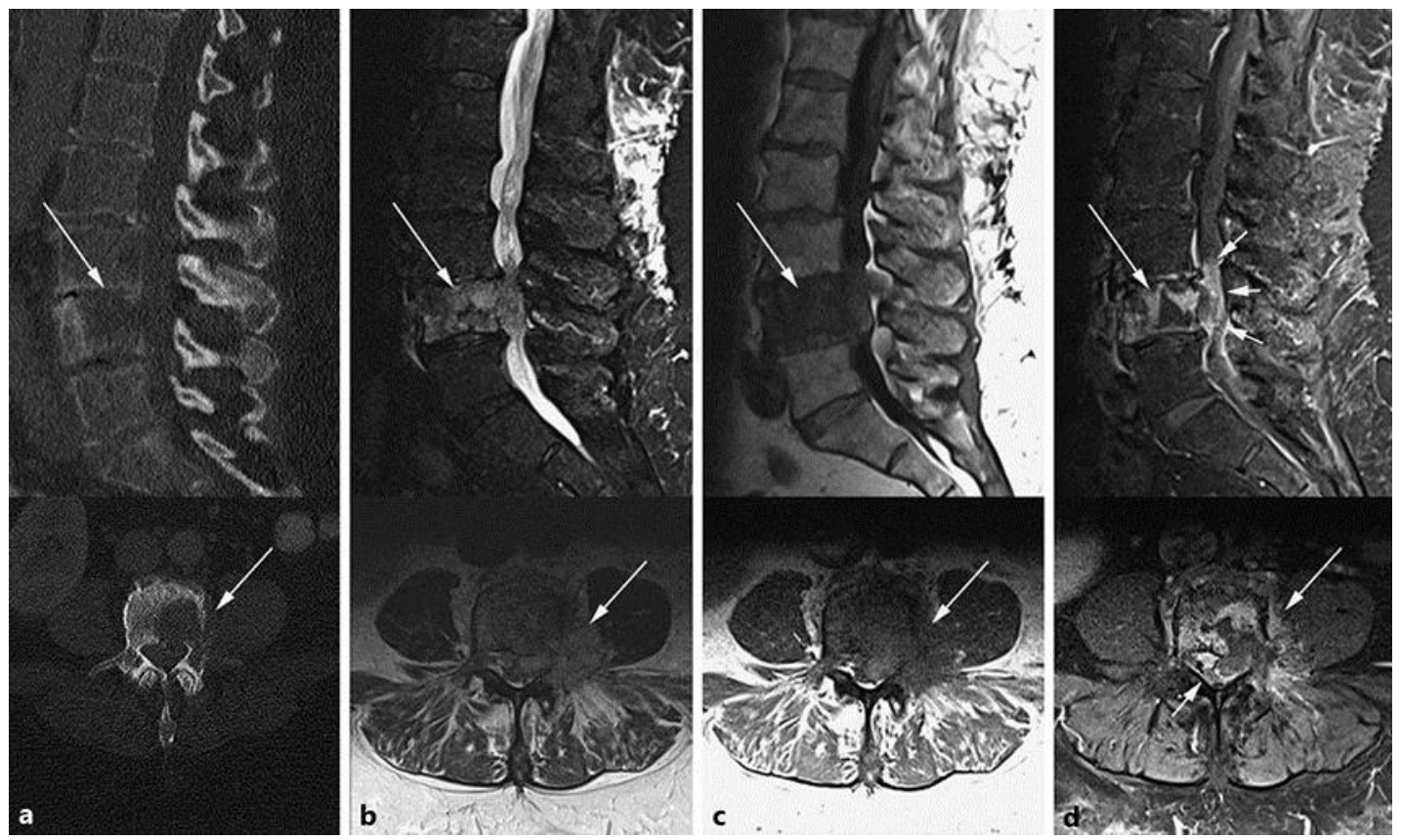

Fig. 3. CT and MRI of the lumbar spine demonstrated an L4 metastasis and pathological fracture of the vertebrae. a Sagittal (top) and axial (bottom) CT images show the lytic appearance of the L4 metastasis (arrow) involving the left posterior vertebral body and the left pedicle. b Sagittal STIR (top) and axial T2W (bottom) images demonstrate the L4 metastasis with epidural and left paraspinal extension (arrow). c, d Sagittal (top) and axial (bottom) T1W precontrast (c) and postcontrast images (d) demonstrate low intrinsic T1 signal and avid enhancement within the L4 metastasis, with both epidural and left paraspinal spread (large arrows). Abnormal enhancement is also present within the cauda equina nerve roots at L4 (small arrows).

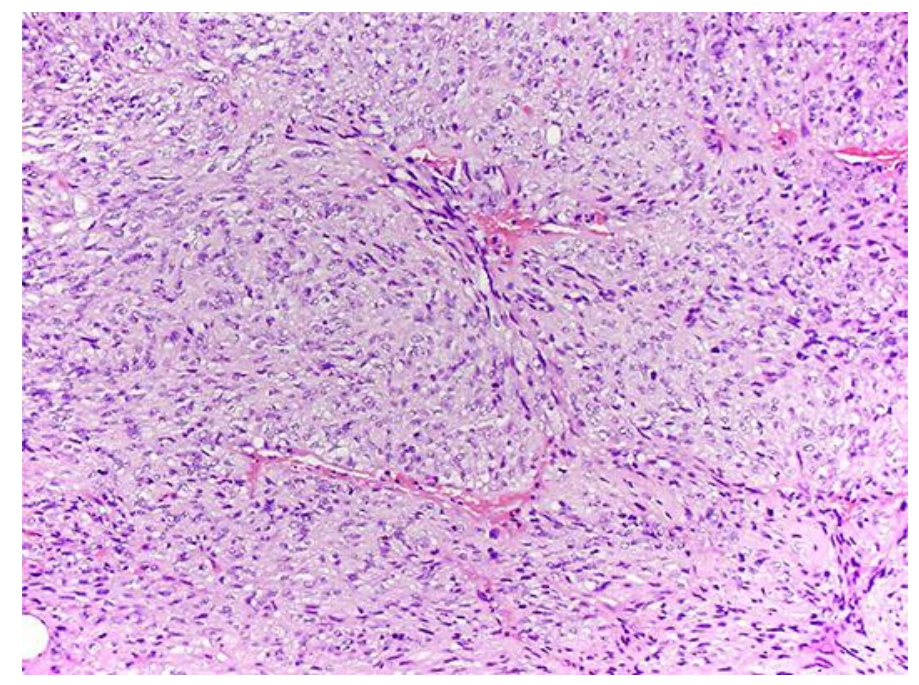

Fig. 4. H\&E stain of the lumbar spine lesion biopsy showing spindle cell sarcoma histologically similar to sarcomatous component of phyllodes tumor. 


\section{Case Reports in Oncology}

\begin{tabular}{l|l}
\hline Case Rep Oncol 2016;9:861-868 \\
\hline DOI: 10.1159/000453660 & $\begin{array}{l}\text { C 2016 The Author(s). Published by S. Karger AG, Basel } \\
\text { www.karger.com/cro }\end{array}$ \\
\hline
\end{tabular}

Johnson et al.: Malignant Phyllodes Tumor Presenting in Bone, Brain, Lungs, and Lymph Nodes

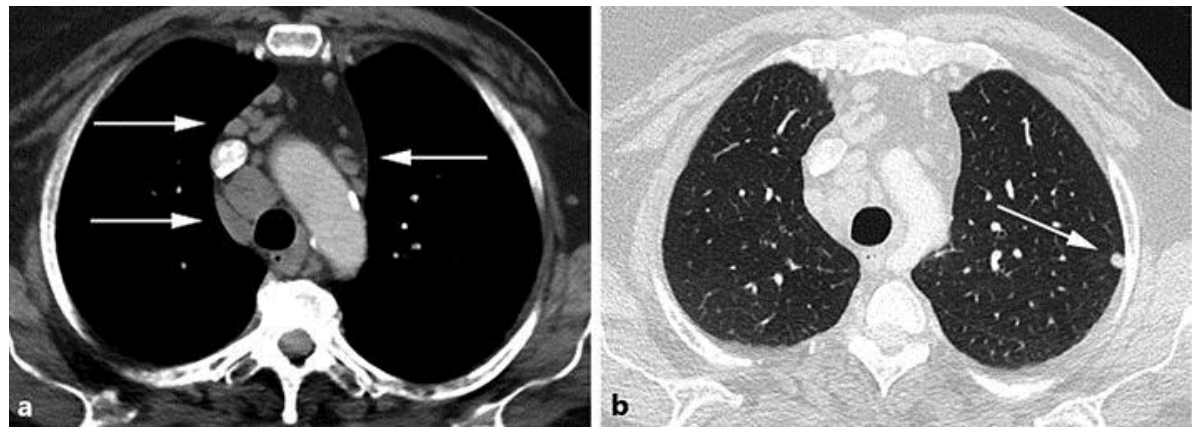

Fig. 5. Contrast-enhanced CT of the chest. a Soft tissue windows show hilar and mediastinal adenopathy (arrows), new compared to 14 months prior. b Lung windows demonstrate a pulmonary nodule in the peripheral left upper lobe $8 \mathrm{~mm}$ in size (arrow), increased compared to the the prior scan and consistent with metastatic disease. Additional subcentimeter nodules are also present, new compared to 14 months prior.
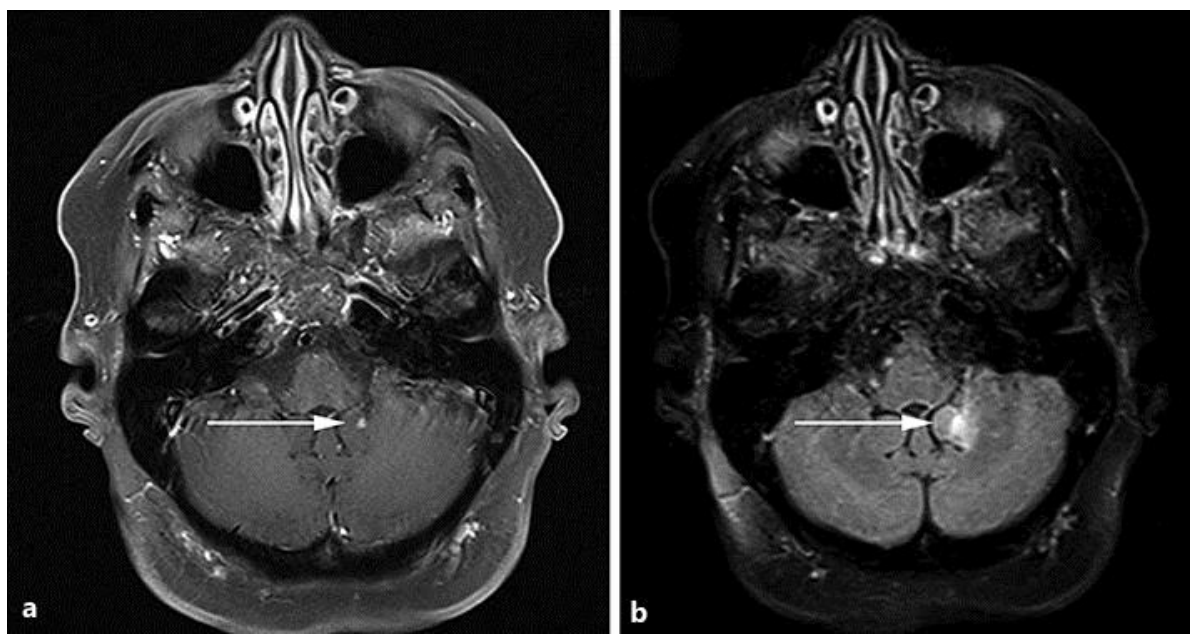

Fig. 6. Contrast-enhanced MRI of the brain demonstrates an enhancing solitary subcentimeter left cerebellar vermis metastasis (a, T1W postcontrast image, arrow) with surrounding edema (b, FLAIR image, arrow). 